\title{
A Semantic Management Model to enable the integrated management of media and devices
}

\author{
Paula Viana · Artur Pimenta Alves
}

Received: date / Accepted: date

\begin{abstract}
The challenge of managing large scale media assets has led to the development of metadata schemas that are expected to enable efficient search and retrieval of multimedia content. Those approaches propose schemas that can range from simple keyword based descriptions to complex hierarchical organization of information. However, effective media asset management requires more than content searching and retrieval: underlying infrastructures are usually complex, require the use of a number of different equipment and management decisions have to be done based on information available from the multimedia metadata layer as well as on data describing system resources and capabilities. In this paper we propose a new ontology that aggregates information from different sources and enables a top level business oriented view of multimedia archives.
\end{abstract}

Keywords Media Asset Management - Metadata Integration - Semantic Metadata Management · Multimedia and Infrastructures Ontologies

Paula Viana

Instituto Superior de Engenharia do Porto

Rua Dr Antonio Bernardino de Almeida 431

4200-072 Porto

Portugal

INESC PORTO

Campus da FEUP

Rua Dr Roberto Frias, 378

4200-465 Porto

Portugal

E-mail: pviana@inescporto.pt, pviana@isep.ipp.pt

Artur Pimenta Alves

INESC Porto

Faculdade de Engenharia da Universidade do Porto

E-mail: palves@inescporto.pt 


\section{Introduction}

The need to easily search, browse and retrieve video content has been growing in the last years due to the rapid evolution of networking and storage technologies and to the requirements for re-using and repurposing content. Professional stuff working in medium and large video producers, as well as in broadcasters, are required to reduce the time of capturing and editing new content and to re-use the company archives to produce new stuff. This new approach to video production and re-purposing led to the growing interest on technologies that would enable functionalities to search, browse and retrieve material available online. Lots of effort has been put in different projects and initiatives for the definition of metadata models: ISO-MPEG7 proposes a generic multimedia information description schema, EBU-P/META and SMPTE, in broadcaster environments, have defined metadata exchange schemas, Metadata Dictionaries and formats for uniquely identifying material, while Dublin Core has been working on a larger range of domains.

One of the open research issues is the definition of methods that will allow the management of system resources in a MAM environment. Effective deployment of such a system in a real environment requires the development of management platforms that contribute to efficient system usage by all the players involved in the archive from the creative people to the technical and administrative manager. Those resource management aspects have not been, up to now, the main concern of researchers and system developers but are a critical issue for the successful exploitation of these kind of systems.

The work presented in this paper enlarges the concept of metadata as it has to be regarded not only as data describing audiovisual content but also as data describing system resources and policies for global resource management. The approach exploits concepts and technologies under development by the multimedia and networking communities and extends these proposals by provided an integrated view of the archive through a domain ontology that integrates knowledge from both worlds.

\section{Related work}

Metadata is data about data, or complementary information about the form and content of a resource. Structured metadata is divided into elements, each of which describes a particular aspect of the information resource. Consistency is important for effective information management and retrieval, so clearly defined standards must be set and widely applied. During the last few years, a number of organizations and individual institutions have been working towards the definition of uniform audiovisual and device description schemas as well as description languages and descriptors. However, metadata is usually not only content but also domain-dependent which means that different sub-sets and variations have been designed for a specific application or usage context or focusing on specific aspects of the production, archiving or distribution process.

An obstacle for the common use of generic standardized metadata models is also that a model often reflects the business model of organizations. From another perspective, when such focus does not exist, standards or description schemes usually grow in dimension and complexity in an attempt to satisfy all the requirements. For these reasons the level of implementation of standardization is limited. 
When dealing with managing large and complex audiovisual archives, metadata refers to the information describing content but also to information describing devices that make up the whole infrastructure.

\subsection{Content Description Schemas}

In an attempt of standardizing content description metadata, numerous organizations and projects proposed different solutions that provide different degrees of granularity.

In the television domain, the first proposals are the FIAT/IFTA Minimum Data List (MDL) [1] by the International Federation of Television Archives (FIAT/IFTA) and the EBU System of Classification of Radio and Television Programmes (ESCORT 2.4) [46]. The first one defines a recommendation used for cataloguing video and film documents and specifies a core set of descriptors divided in three groups: identification (title, date, producer), technical data (keywords, content, format, language), rights management (origin of the material, contracts, copyrights). The second proposal defines a classification scheme to describe not only the form and content of radio and television programs but also the way they were produced, scheduled and transmitted, how they were received by the audience and the costs involved.

The EBU P/Meta Scheme [6,5] defines the attributes and transaction sets that have been identified to support the exchange of media assets between EBU members as content creators, distributors and archives. It supports the exchange of metadata - with or without content - based on agreed definitions of Attributes, Attribute Type values, Sets of Attributes, Sets of Sets, unique identifiers and protocols in scenarios of Business-to-Business (B2B) and System-to-System (S2S) transactions. Exchange of metadata associated to essence can be done in several ways: isolated, embedded into streams or encapsulated within files, using for that external standards as XML, SDI, MPEG2, MXF, etc. Although the purpose of the P/META Scheme is to support the exchange of content between trading entities, and is not directly associated with schemas designed to model storage, its attributes must map to theirs when items go into the archive.

Still in the television domain, SMPTE defines a set of descriptors [7] covering different aspects of the production chain. Metadata elements are grouped in a small set of classes ( Identification, Administration, Interpretation, Parametric, Process, Relational and Spatio-temporal) each representing a collection of metadata elements with common characteristics. Within this schema, metadata elements are organized into a hierarchical structure and cover aspects that range from technical information on video/audio encoding characteristics, to information on how the essence was processed/manipulated, who was the supplier and what are the rights. Interpretive information like a narrative summary or a title is also provided.

Another solution initiated in an industrial forum is the standard known as TVAnytime (TVA) [8]. This standard defines not only a metadata schema able to describe content to be delivered to a set of different terminals (mobile phones, PDAs, personal video recorders, etc), but also the architecture and communication aspects to be considered when implementing a TV-Anytime compliant system. TV-Anytime has defined a unique document structure to aggregate programme descriptions, user descriptions or classification schemes (e.g. Content/genre, Action-Type, ContentCommercial, HowRelated, TVARole). A TVA system is articulated around the Content 
Referencing Identifier (CRID). A CRID relates to a specific content/programme instance, or to other CRIDs themselves referring to other different instances. The CRID is also the link between a content instance and its associated metadata. To enable some flexibility, TVA uses XML as the representation language and allows the refinement of the descriptions by adding extra information.

MPEG-7 (Multimedia Content Description Interface) [2-4] provides standardized content description for various types of audiovisual material and is not intended to be used in a particular area but to be applied to such distinct applications as surveillance, home entertainment, broadcast channel selection, educational applications, etc. The standard defines descriptive elements that range from very 'low-level' signal features like colours, shapes and sound characteristics, to high-level structural information about content collections; a Description Schemes (DS), that specify the structure and semantics of the relationships between their components and a Description Definition Language (DDL) to define the syntax of the MPEG-7 Description Tools.

Although its original intention was the representation of bibliographical information on the web, the Dublin Core Metadata Initiative (DCMI) [9] proposes a metadata standard that supports a broad range of purposes and business models. It has evolved to a standard that aims at allowing the creation of simple descriptive records for any information resources (e.g., electronic documents, JPEG images, video clips). The DCMI defines a set of 15 main descriptors organized in three main sets related to different aspects: Content, Coverage, Description, Type, Relation, Source, Title, Intellectual Property, Contributor, Creator, Publisher, Rights, Instantiation, Date, Format, Identifier and Language.

Although each of these initiatives claims to address different objectives and cover different areas of the business, interoperability and mapping between different namespaces would have to be obtained in order to enable a transparent access to different content providers.

\subsection{Device Description Schemas}

The problem of information description is also important in the domain of device an network management and metadata schemas have also to be defined to enable uniform access to information.

One of the most popular solutions is SNMP (Simple Network Management Protocol) [10] which defines not only a protocol for information access but also the structure of the information base - the MIB (Management Information Base) [11]. Although SNMP was mainly driven by the network community and the initial targets were TCP/IP routers and hosts, many manufacturers of multimedia equipment have defined private MIBs for managing their devices. The main strength of SNMP is its simplicity that contributed to a widely adoption of this framework.

Until recently, storage devices have been managed and controlled by a single application installed with them. The shift to networked architectures, where storage devices and hosts are connected to networks interconnected by routers and switches, brought the possibility for each host to access each storage device increasing the complexity of the system. One of the problems introduced by this new scenario was the lack of a standard that isolates the applications from the storage hardware, implying adaptation of devices to existing applications or rebuilting of applications to support new hardware. To solve this problem, IEEE proposed the Media Management System (MMS) 
[12] which provides a data model for typical storage devices like libraries, cartridges or drives.

A wider application solution is provided by the Web Based Enterprise Management (WBEM) initiative that applies to all manageable resources available in a network. Its main goal is to define a Common Information Model (CIM) [13] that describes various aspects of the management environment. The schemas are provided in UML, XML and MOF (Management Object Format), a text based language.

All these approaches result in a multiplicity of languages and data models with different complexity, extensibility and applicability. The problem of mapping the different data models is similar to the previously described for the multimedia content description approaches - pair wise mapping between each of the descriptors is needed.

\subsection{Ontologies in Management Solutions}

Although XML [15] and XML Schema [14] have been widely used to represent metadata, as they enable syntactic interoperability through data parsing, they fail in providing semantic interoperability as a great part of the semantics remain implicit. Therefore, each time an application is developed, semantics must be extracted from the standard and re-implemented. Introducing new languages that fill the semantic gaps of XML has to be considered. In summary, the following characteristics can be assigned to each of the available languages:

- XML and XML schema - XML provides a mean to represent a structured document without imposing any semantic constrains while XML schema enables constraining the XML document structure. However, querying a XML database in order to retrieve e.g. an MPEG-7 SegmentType description, requires that the application is aware of the hierarchy of segment types i.e. VideoSegmentType, AudioSegmentType, etc. [26]

- RDF and RDFS (RDF Schema) - RDF [17] is built upon XML and XML Schema and aims to provide a model that enables the description of the documents' semantic using the concept of resource and relations between those resources. RDFS [16] enriches RDF by enabling the definition of classes (Class) and properties (Property), range and domain constrains on properties, subclasses (subClass0f) and subproperties (subPropertyOf) allowing the specification of an hierarchical organization of classes and properties.

- OWL (Web Ontology Language) - OWL [18], layered on top of RDFS, adds capabilities to RDFS. It adds various constructors for building complex class expressions, cardinality restrictions on properties and mapping between classes and individuals such as equivalentClass, sameAs, inverse0f, etc [19]. Reasoning is made possible as new knowledge may be inferred e.g. by transitivity. Querying an ontology doesn't require the user to have a global knowledge of the ontology schema.

It is clear from this summary that OWL enables greater flexibility when dealing with different metadata schemas as it provides richer descriptions that allow the formalization of a domain and enable automated reasoning support. Adopting OWL for coding the management information was proposed by a set of authors in different domains as identifies below. 


\subsubsection{Multimedia Ontologies}

One of the first publications that describes the need for the semantic description of audiovisual information is [20]. In this paper, the author highlights the drawbacks of using XML representations of MPEG-7 and MPEG-21 as, although the XML Schema language provides support for explicit structural, cardinality and data type constraints, it provides little support to enable efficient and flexible mapping and integration of different schemas as the semantic knowledge needed is not available. The use of ontologies and formal machine-processable representations such as RDF Schema or OWL is proposed. Given that overlaps, redundancies and incompatibilities between the semantics of the terms used both in MPEG-7 and MPEG-21 were detected, the author proposed the definition of a top level core ontology that would contribute to the easier integration of MPEG-7 and MPEG-21 information.

A similar approach is proposed in [21] and [22]. The work presented proposes the use of MPEG-7 XML Schema for expressing the structural meaning of audiovisual documents and OWL/RDF for expressing the ontological meaning of the constructs. It also details a core ontology that contains all the concepts and relationships necessary for a wide range of applications. The terminology used is oriented towards the description of radio and TV programmes and uses concepts from MPEG-7, TV Anytime and IPTC (through the ProgramGuideML initiative). The use of constructs not available in XML schema enable e.g. the definition of a StudioProgram as exactly an HomogeneousProgram whose all sequences are StudioSequences (a set of concepts defined in the ontology). Expressing basic axioms such as "a TVProgram cannot be both a HomegeneousProgram and a HeterogeneousProgram" is also made possible through the use of the disjointWith OWL construct.

A methodology to enable the interoperability of OWL with the MPEG-7 schema is provided in [23]. This approach enables domain ontologies described in OWL to be transparently integrated with MPEG-7 metadata meaning that applications that recognize and use MPEG-7 constructs can make use of domain ontologies for applications like indexing, retrieval, filtering, etc. In [24] and [25] the same authors extend this approach to include TV-Anytime metadata.

The work presented in [26] provides a complete and automatic mapping methodology of the MPEG-7 standard to OWL. It extend the previous solutions by providing a systematic approach that can be applied to the whole standard. A generic XML Schema to OWL mapper combined with an XML to RDF translator is used. This method has also been applied with other XML schemas in the Digital Rights Management domain $[27,28]$ and in the journalism domain [29].

An MPEG-7 based multimedia ontology, composed of multimedia DOLCE (Descriptive Ontology for Linguistic and Cognitive Engineering) based patterns is proposed in [30]. The main objective is to enable web interoperability and to describe multimedia resources on the web.

The proposal described in [48] argues that although linguistic terms are appropriate to distinguish event and object categories, they are inadequate when they must describe specific or complex patterns of events or video entities. To bypass this limitation, an enriched ontology that uses visual prototypes (either images or video clips) that capture the essence of the event or entity is proposed. High level concepts, expressed through linguistic terms, and patterns specification, represented by visual prototypes, are then organized into a new ontology where images or video clips are added to the ontology as specification of linguistic terms. The proposal is tested within the soccer video domain. 
Some work has also been developed in order to bridge the gap between low level visual descriptions and high level domain concepts. The tool described in [49] allows linking low-level MPEG- 7 visual descriptions with ontologies and annotations.

\subsubsection{Network Management Ontologies}

Research in the domain of network management found similar problems. Although the use of XML was formerly applied for managing network devices [32,31], the lack of semantics in the data models led some authors to try to use ontologies. In [33] the authors discuss the use of ontology languages for describing management information and compares OWL to standard approaches like SMIv2 or MOF/CIM. Some examples of the translation to OWL of the CIM_ManagedSystemElement are provided. The analysis shows that ontology languages enhance the semantic expressiveness of the definitions and a set of tools that can be used to validate and reason with them are identified.

Methods to formalize the Common Information Model (CIM) metaschema are proposed in [34] and [35]. The authors claim that CIM models are specified using a semi-formal ontology that limits its ability to support important requirements of a self-managing distributed system including knowledge interoperability and aggregation, as well as reasoning. To support these requirements, they propose a framework for constructing a CIM ontology based upon mappings from Unified Modelling Language (UML) constructs to ontology language constructs. Advantages and drawbacks of this approach when compared to the direct translation of all management information into OWL are presented.

\section{A Domain Ontology for Media Asset Management}

\subsection{Characterization of the Problem}

As discussed in the previous sections, a number of metadata models have been proposed in order to allow the implementation of important tasks in media access and system management. However, due to the complexity of the problem, most approaches pick up a particular problem. The initiatives described above cover aspects directly related with device management (disk, tape libraries, network devices, etc.) and define management metadata without relating the system with the application area. On the other side of the line, archivists have been mainly interested on the definition of a metadata schema that enables the process of searching and locating items in their huge assets.

These two working areas are not dealing with the problem of aggregating descriptive metadata with management metadata to allow an integrated view of the archive and to avoid data inconsistency in the two different layers.

The need for information integration from different domains, when dealing with aspects of managing a complex audiovisual archive, can be better illustrated by the presentation of some examples that provide simple cases of such synchronization requirements:

Example 1 Suppose a programme that is archived in two different files (for example, part 1 and part 2 of a news magazine). For the client applications (searching and editing workstations), due to metadata introduced in the descriptive archive by the annotation workstation, the program is seen as 
just one stream. For the management application, they are however different files, possibly with different number of accesses. Using inactivity as the criterium for moving material to a secondary storage device (e.g. a tape library) in an hierarchical storage management approach, would introduce an unwanted difference between the two files of the same programme. Migration of content to and from a secondary storage system requires then information not available from a single metadata layer;

Example 2 Although in most cases the master tape is not circulated, because there are browse copies as VHS tapes, when the full-quality material is needed, the unique archive item has to be taken out of a controlled environment and exposed to a degree of risk. In old assets like television or historical archives, the amount of material with recycling needs is large and the prioritisation of tape transcription due to preservation needs depends on tape characteristics available in the Storage Resource Management (SRM) system, including number of times it was mounted, read and written, but also on descriptive information classifying a program. This last information is only available from content description metadata. None of the isolated metadata solutions is able to provide an answer and the integration of the two domains is then needed to provide the libray manager a list of tapes with different levels of priority concerning transcription of material;

Example 3 Technology obsolescence may also dictate the need for content migration. Since the introduction of videotapes for archiving TV programme material, some attention has been put on the need to properly handling tapes. It is commonly accepted that well archived tapes can last for 20 or 30 years. Besides tape longevity, archivists have also to deal with replay equipment lifetime. The total lifetime of a video tape rarely exceeds 25 years including the production period and the manufacturers support after the termination of product availability. Although replay capability of a format is sometimes prolonged by the compatible replay of its successor format, it is already very dificult to find equipment for reading some of the oldest formats: 2-inch Quad, 1-inch B, 1-inch C and Umatic are strongly endangered and Betacam, Betacam SP and M-2 formats are expected to be endangered in a near future. The hardware available for tape reading, information obtained in the SRM metadata, may then be crucial for defining the prioritisation of recycling: if the number of devices still able to read a certain video format is low, material should be transcripted to a new format. However if the number of items needing recycling is huge, some extra criteria has to be used to provide a resonable list. Once again, the final decision shall be based on the genre of the programmes affected or in other content characteristics. The answer to this recycling need requires again the integration of two sources of information;

Example 4 Most architectures proposed to provide online access to video libraries suggest the use of different qualities of the material: besides the full quality, different browse versions are usually available in the system. If this happens and different quality versions of the same content are available, the network capacity and availability, information coming from SRM metadata, may determine which version should be sent to a user. Content description information associating different versions to a single programme item is then needed; 
Example 5 Given that storage costs depend on the support device, different types of archives are usually used: online archives that enable fast access but have higher costs and near online archives based on robotic solutions which enable lowering the costs but require extra time to access content. Stream migration between these different type of archives depends on the disk capacity available from the SRM system but also on description information aggregating video, audio and key frames files available from content metadata. File access logs available from the SRM system may also provide essential information concerning important material that has been rarely used and should be highlighted.

Analysing the different examples presented, two conclusions can be immediately highlighted: 1) the integration of the two metadata domains will provide extra information and 2) there is the need for another metadata plane that shall define a set of rules that enable business or high-level management decisions:

- Migration between archives is not only dependent on disk capacity available on a server but also on the percentage that a manager wants to have free;

- Prioritisation of re-cycling has to be done not only based on tape preservation aspects but on a policy defining the importance of the items contained;

- Renewing the rights of use of a given programme depends on the costs and on the current or expected value of that content;

- Available bandwidth may have to be reserved based on the editorial constrains.

Given the size of some historical assets, such as national broadcasting archives, their social and economic value and their preservation problems, these examples clearly demonstrate that some crucial tasks of a management application are not easily implemented using the available metadata information as 1) it is too complex for the purpose of management, 2) no integration of the different models is provided, 3) some business-driven management related metadata is missing and, finally, 4) it is difficult to have specialized personal with knowledge in all the areas.

The work presented in this paper proposes the integration of those metadata planes and is expected to enable a step ahead on Media Asset Management solutions in complex environment. The definition of a domain ontology that captures and provides a formalization of the problem and enables the description of different aspects that affect management is presented.

\subsection{Ontology Conceptualization}

Despite the variety in metadata models, all the specific proposals share common concepts and properties when describing audiovisual documents or devices. For instance, the concepts of Genre or some Production and Broadcast properties (Target Audience, Broadcast Date, Live or Pre-recorded Broadcast) are always needed either for cataloguing and indexing or to parameterize an algorithm whose goal is to extract automatically some features from the signal.

Another aspect that is present in most of the description schemas is the Credits List, that is, the people and their roles in the programme. Technical activities, such as Direction, Production, Pos-production (Special Effects, Captions, etc), or people 
actually participating in the programme as Actors, News Readers or as Interviewed Guests can be used as key information concerning the management of the archive.

Example 3 presents one of the problems that the most important archives face: media obsolescence. Migration to a new support requires the manager (either a person or a software application) to be aware of the media format of a given audiovisual object. Besides the genre or the actors involved in the programme, prioritization on migration may be determined by a set of factors where the programme award list may be important.

After defining precisely the concepts required, the next step is to introduce relations between the different entities in order to structure the knowledge and enable reasoning. Taking into account the examples, some relations can be elicited: 1) relations between the audiovisual objects and people (hasProducer, hasParticipant), 2) relations between the audiovisual object and the broadcast properties (hasAudience, isLive, wasBroadcastedAtTime), 3) relations between the audiovisual object and technical features (hasFormat, hasRights) and 4) relations with external ontologies that identify e.g places.

The main concern on building this ontology was to integrate, in a unique view, information from the different domains giving the manager an upper view of the main aspects that might affect management: the AudioVisualObject and the ManagedElement are the two main classes of this ontology and enable, respectively, the description of content and of the systems to be managed based on some criterion to be defined. Auxiliary classes (Person, Property) were added to enable additional information to be infered from the ontology. Extending the ontology can be done by adding new classes (e.g. Place) to the current version. Figure 1 presents a graphical view of the first two levels of this ontology that will be detailed in the next sections.

\subsubsection{The AudioVisualobject}

This ontology distinguishes audiovisual content based on two concepts: their processing status (either a final Programme or Rushes coming directly from the camera without editing) and their level of importance (e.g keyframes, audio tracks, etc. are considered to be OtherMedias that might be associated to a Programme through an has-associatedMedia association but that are not the main object of management).

The AudioVisualobject is defined as a superclass that aggregates these three concepts and has a set of generic properties (title, synopsis, etc.) that are inherited by the subclasses. The characterisation of the object Programme requires the definition of a set of other properties (has-genre, has-team, has-targetAudience, etc.) that provide extra information on the content and that are available from most of the multimedia description schemas.

\subsubsection{The ManagedElement}

Management operations are to be performed over systems that make the archive operational. High level decisions, as the ones presented in the examples, will be based not only on the characteristics of the content but also on the hardware availability and its characteristics. The ManagedElement is used to describe manageable hardware or physical resources: 


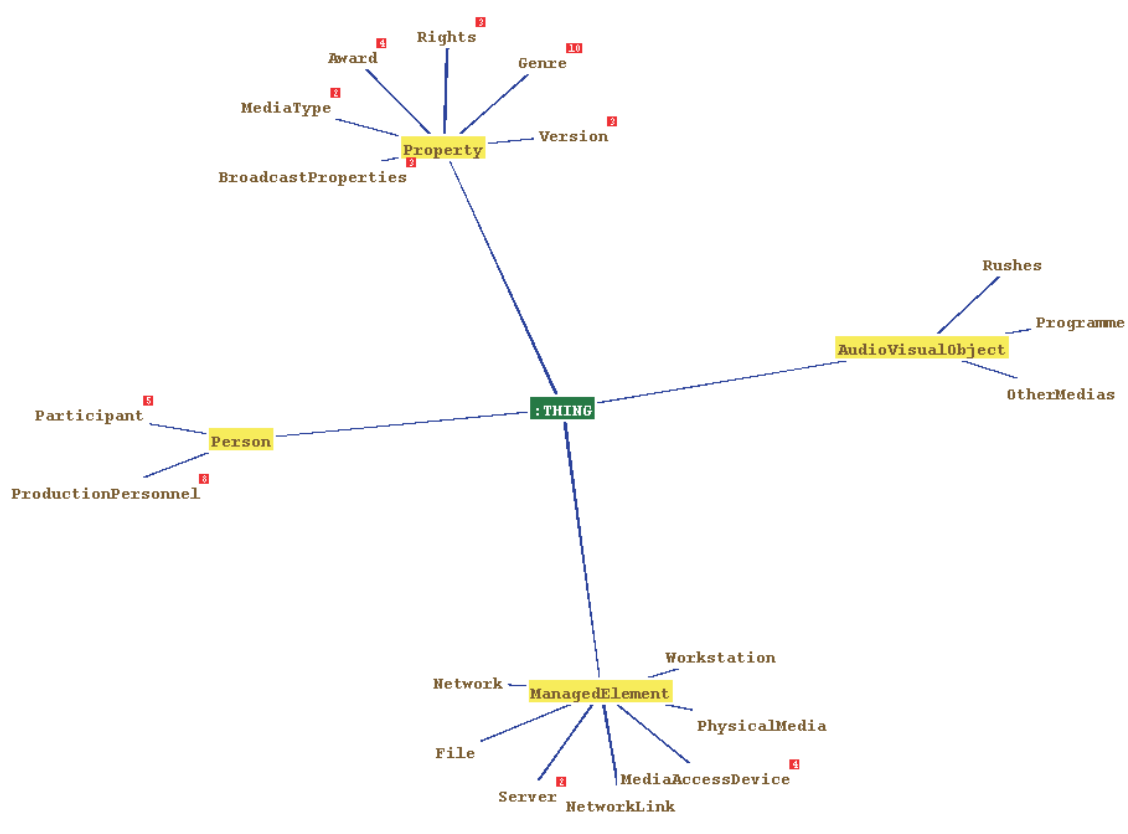

Fig. 1 Partial view of the top levels of the Management Ontology

- The MediaAccessDevice class tries to catch the characteristics and manageability of all types of media access devices. A MediaAccessDevice represents the ability to access one or more media and use this media to store and retrieve data. This class has a set of subclasses that concretize the type of media the device is able to read (e.g a 16mm film, a 1" tape, a Betamax tape, a Umatic tape, etc.). This class can be directly mapped to the CIM:Device:MediaAcessDevice class. A set of properties from this CIM class were selected based on its importance concerning high level management decisions.

- The object of management in an archive is the content that is stored in tapes and films. The class PhysicalMedia, that can be directly mapped to CIM:Physical:PhysicalMedia, represents any type of storage medium. This class is typically used to locate and manage removable media. It describes the main properties of those physical supports that might affect e.g. recycling priority (has-mediaType, maxMounts, mountCount, etc.).

- The archive is deployed in a media area network requiring, then, the identification of each subnetwork (the class Network in this ontology). From the point of view of the high level management, this information is not directly relevant but will be required by the system for reasoning on the network load on the link that connects a Workstation and a Server.

- The NetworkLink class is a simplified version of the CIM specification for characterising network connections. From a business point of view, the manager is not interested in details of the technology but mainly on the aspects that enable an efficient management of the resources. This class re-uses concepts from the CIM:Core:ActiveConnections association and from the CIM:Network:Protocol- 
EndPoints and CIM:Network:NetworkPipe classes as well as from other specific classes concerning communication technologies used. Each instance of this class represents a connection between two instances of a Network and includes information such as the status of the link, the totalBandwidth, the ReservedBandwidth or the activeBandwidth. The association connects is used to identify the two endpoint.

- The Server class catches the characteristics of the essence archives: the On-Line Archive (OnlineServer) and the Near-On-Line Archive (NearOnlineServer). A set of properties enables the identification of the location (is-ConnectedTo), characterisation of the storage capacity (TotalManagedSpaced and RemainingManagedSpace) as well as the definition of some management requirements like LowSpaceWarningThreshold. The OnlineServer maps to the CIM:Device:StoragePool class.

- Activities like annotation, browsing and editing are performed in a Workstation that is-ConnectedTo an instance of a Network.

- The File class is used to relate content available in an OnlineServer with an Audiovisiualobject through the property has-file. The association is-availableFrom identifies the Files available from a Server.

An example of the associations between the classes ManagedElement and AudiovisualObject is presented in Figure 2. This view enables, for example, the association between an Audiovisualobject and a MediaAccessDevice that is able to read it through the associations has-copy, is-stored-in, has-mediaType and is-Read-by.

\subsubsection{The Property}

AudioVisualObjects will have a set of properties that enable additional information to characterise content. The main concepts that can be found on this branch of the hierarchy are:

- A classification of the content according to a set of themes (the Genre class). The taxonomy defined for this ontology can easily be mapped to TV-Anytime ContentCS [45], EBU-Escort2.4 [46] and DVB-EIT [47] classification schemes. A Programme can be catalogued e.g. as NonFiction/ Information, Drama/Fiction, Amusement/Entertainment/Hobbies, Music/Ballet/Dance/Art or Sports or, if more detailed information is wanted, as any subclass of these classes.

- In a television environment, information concerning broadcast (e.g. BroadcastMode and TargetAudience) is available under the BroadcastProperties class. Once again, for enabling an easy mapping with other ontologies, TV-Anytime and EBUEscort2.4 were used as the basis for the target audience classification scheme (for example, GeneralAudience and AgeGroups are available classes with a set of subclasses that follow the existing taxonomies to classify the public).

- Decision e.g. on the need for preservation of a given content might be influenced by the list of awards that a Programme has. An association has-award between the classes Programme and Award is possible.

- The purchase type (PlayForEver, PlayforPeriod, PlayCounts) can help the manager to signal content that should be re-used due to the fact that the owned rights are expiring. The hierarchy defined for the Rights class follows the classification scheme of the TV-Anytime standard.

- Given that content can be available in different quality versions, the class Version reflects this classification and might be used, together with information concerning 


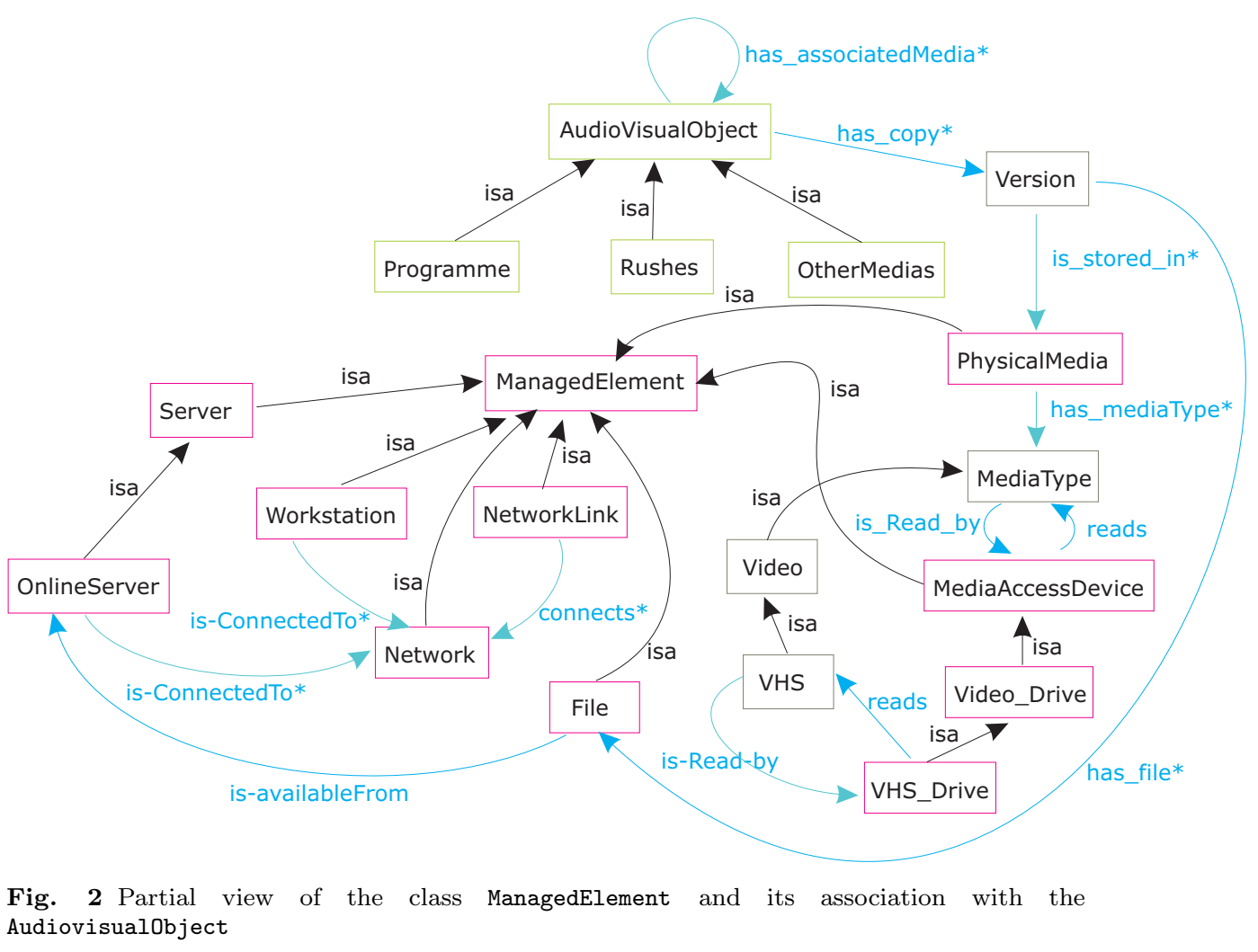

network load, to optimize resources (see Example 4). The slot has-copy enables the association of the classes AudioVisualobject and Property:Version.

- MediaType is an abstract class used to classify PhysicalMedia based on the type of tape/film used to store the content.

A partial view of the class Property and its subclasses is presented in the tree of Figure 3. To enable an easier reading, not all the hierarchy is presented in the picture.

\subsubsection{The Person}

The last part of the ontology contains the concepts that enable a Person to be associated with a Programme based on its role: technical staff can be identified through the class ProductionPersonnel (which can be further classified e.g as a Director or ScriptWriter) while a participant in the programme is identified through the Participant class (which can also be specialised e.g as an Interviewer or an InvitedGuest). This class can be mapped to the TVA:RoleCS [45] and MPEG7:CS.RoleCS.

Figure 4 presents a partial view of the hierarchy of the class Person and the relation of the class Programme with the top level classes.

Examples of some of the concepts defined in the ontology are presented in Tables 1,2 and 3. A complete version of the ontology is available in [44]. 


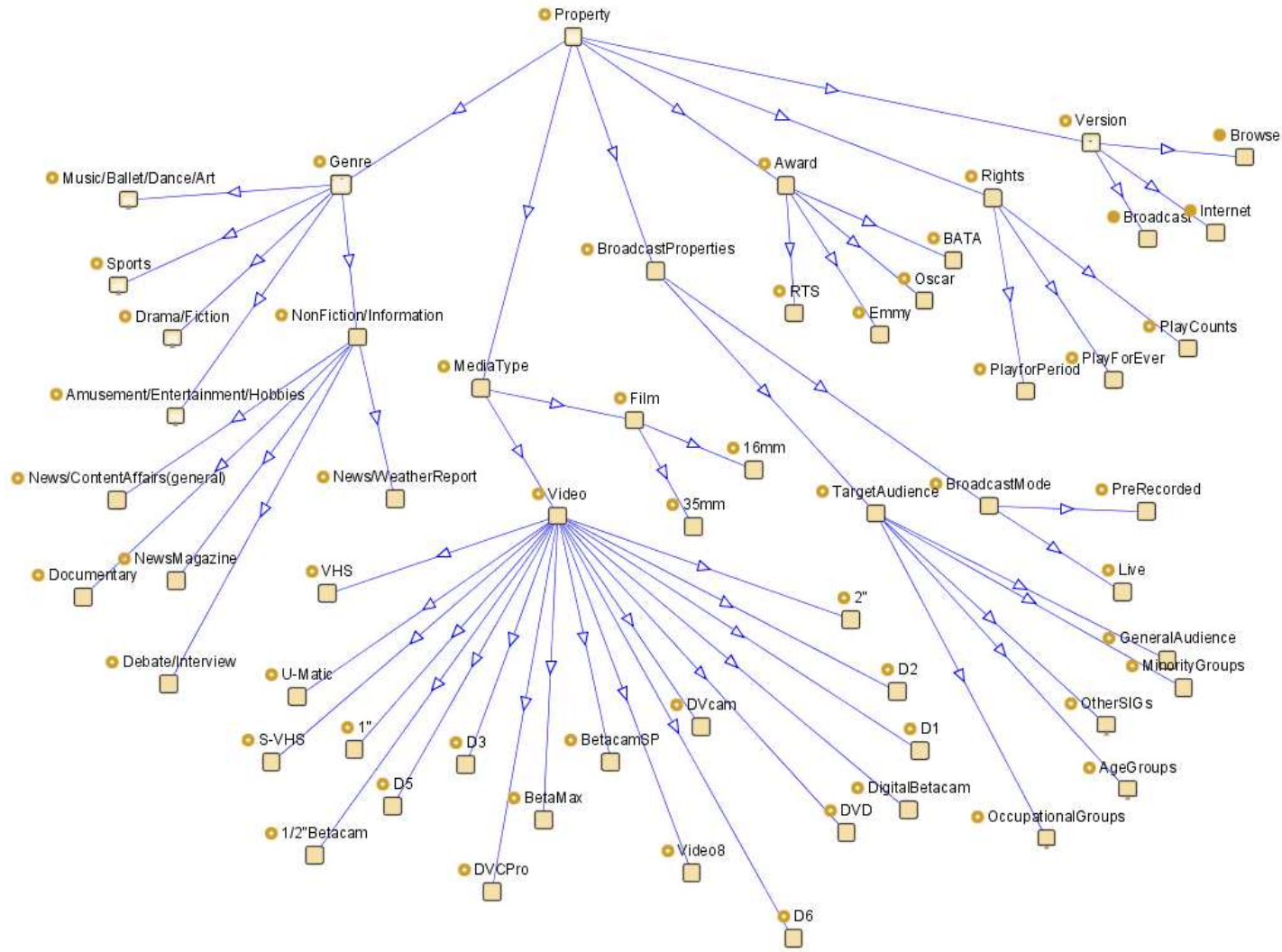

Fig. 3 Partial view of the class Property

Table 1 Four main classes of the management ontology

\begin{tabular}{lll}
\hline Class & SubClasses & DomainOf \\
\hline AudioVisualObject & $\begin{array}{l}\text { Programme, Rushes, } \\
\text { OtherMedias }\end{array}$ & $\begin{array}{l}\text { has-associatedMedia, } \\
\text { has-copy, productionDate, } \\
\text { synopsis, timeOfLastAccess }\end{array}$ \\
ManagedElement & $\begin{array}{l}\text { File, MediaAccessDevices, } \\
\text { NetworkLink, Network, } \\
\text { PhysicalMedia, Server, }\end{array}$ & \\
& Workstation & \\
Property & ProductionPersonnel, & name \\
& $\begin{array}{l}\text { Participant } \\
\text { Genre, }\end{array}$ & \\
& $\begin{array}{l}\text { BroadcastProperties, } \\
\text { Award, Rights, Version, } \\
\text { MediaType }\end{array}$ & \\
\hline
\end{tabular}




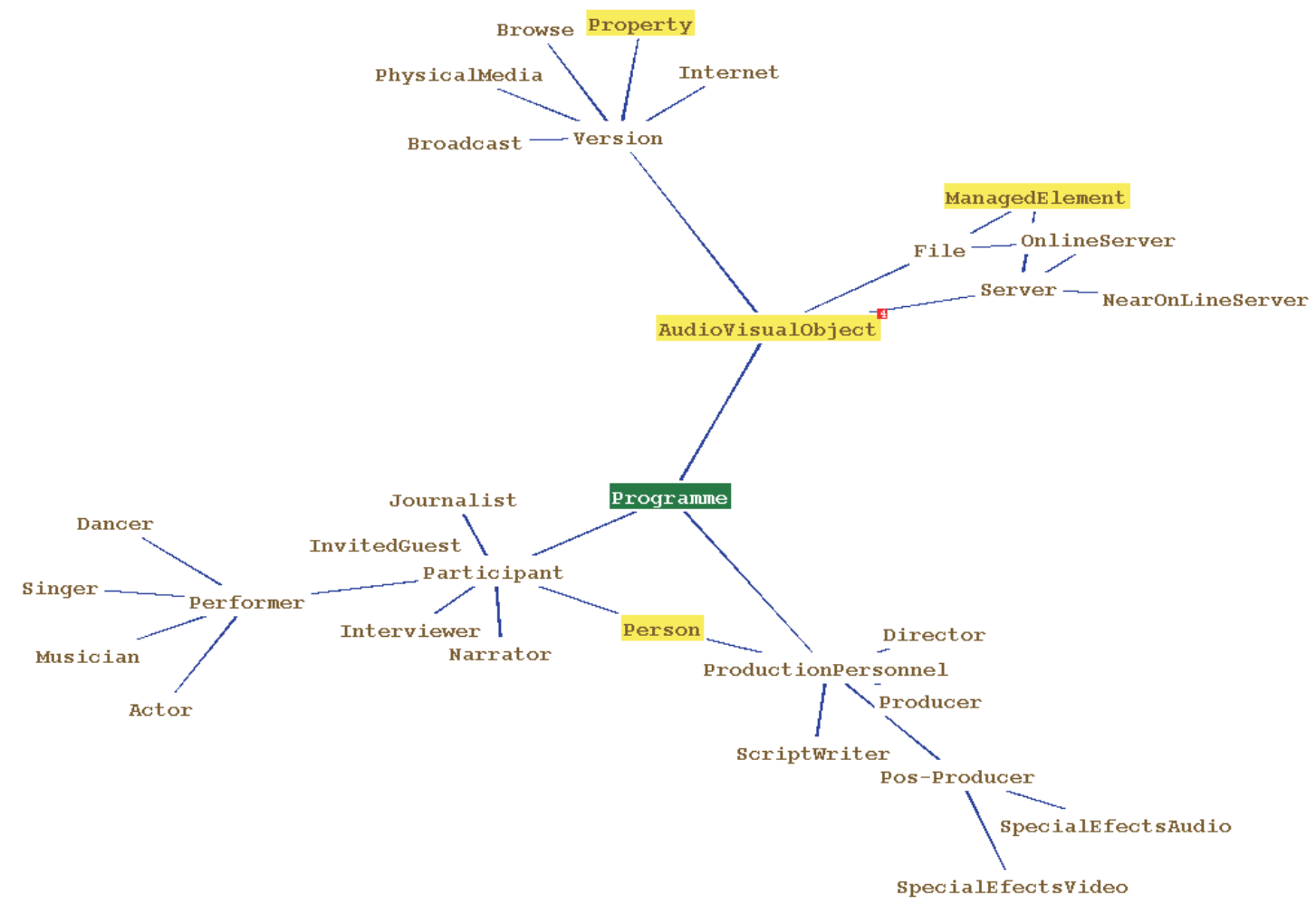

Fig. 4 Partial view of the class Programme and its association with the top level classes

Table 2 Device Management related Classes

\begin{tabular}{|c|c|c|}
\hline Class & SubClasses & DomainOf \\
\hline MediaAccessDevice & Film_Drive, Video_Drive & $\begin{array}{l}\text { lastCleaned, loadTime, } \\
\text { maxAccessTime, mountCount, } \\
\text { name, needsCleaning }\end{array}$ \\
\hline PhysicalMedia & & $\begin{array}{l}\text { has-mediaType, } \\
\text { lable, maxMounts, } \\
\text { mountCount, timeOfLastMount, } \\
\text { totalMountTime }\end{array}$ \\
\hline NetworkLink & & $\begin{array}{l}\text { activeBandwidth, connects, } \\
\text { linkID, reservedBandwidth, } \\
\text { status, totalBandwidth }\end{array}$ \\
\hline Video_Drive & $\begin{array}{l}\text { 1_2_Betacam_Drive, 1_Drive, } \\
\text { 2_Drive, BetacamSP_Drive, } \\
\text { BetaMax_Drive, CDROM_- } \\
\text { Drive, D1_Drive, } \\
\text { D2_Drive, D3_Drive, } \\
\text { D5_Drive, D6_Drive, } \\
\text { DigitalBetacam_Drive, } \\
\text { DVD_Drive, SVHS_Drive, } \\
\text { Umatic_Drive, VHS_Drive, } \\
\text { Video8_Drive }\end{array}$ & reads \\
\hline
\end{tabular}


Table 3 Media related Classes

\begin{tabular}{lll}
\hline Class & SubClasses & DomainOf \\
\hline Genre & $\begin{array}{l}\text { NonFiction/Information, } \\
\text { Drama/Fiction, Amusement/- } \\
\text { Entertainment/Hobbies, } \\
\text { Music/Ballet/Dance/Art, } \\
\text { Sports } \\
\text { Interviewer, Journalist, } \\
\text { Performer, InvitedGuest, } \\
\text { Narrator }\end{array}$ & \\
Programme & participates-In \\
& & $\begin{array}{l}\text { broadcastDate, } \\
\text { has-audienceShare, has-award, } \\
\text { has-broadcastMode, has-genre, } \\
\text { has-participant, has-rights, } \\
\text { has-targetAudience, has-team }\end{array}$ \\
\hline
\end{tabular}

Table 4 Definition of the concept Programme using OWL

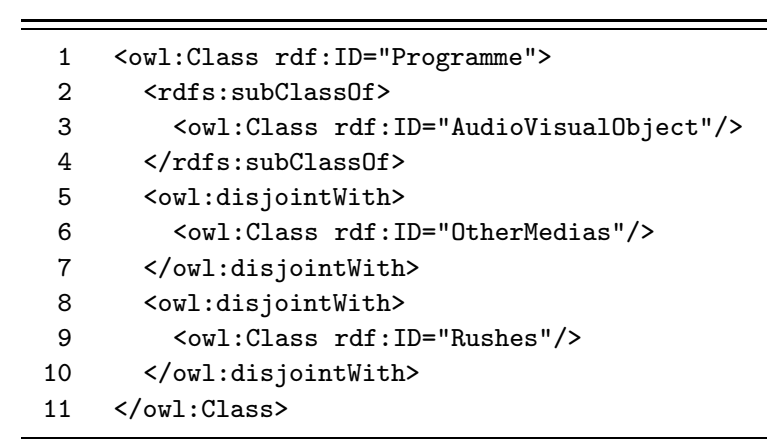

\subsection{Ontology Coding}

Given that XML doesn't provide all the constructors needed to formally express the ontology, OWL was used to code the Management Ontology. This enables the definition of formal semantics for the concepts modelled in the previous step.

One example of the use of OWL constructs is on coding the concept Programme, defined as a subclass of the class AudioVisualObject. To assure that a piece of content is not classified both as a Programme and as one of the other subclasses of the AudioVisualObject class, the construct disjointWith was used. Table 4 defines these concepts using OWL.

Video drivers (class Video_Drive) are one of the objects of management in an archive. Drivers are classified according to the technology implemented and defined as sub-classes of the hierarchy ManagedElement:MediaAccessDevice:Video_Drive. Each type of drive can read only one type of tape and this information can be crucial for decision making concerning tape migration due to lack of reading equipment. This aspect was coded using the OWL mechanisms for adding constrains to a property by declaring it as 'functional'. Table 5 shows the use of the OWL term owl:FunctionalProperty and owl:hasValue to declare that a Umatic_Drive reads only UMatic tapes. 
Table 5 Restrictions on the property reads using OWL

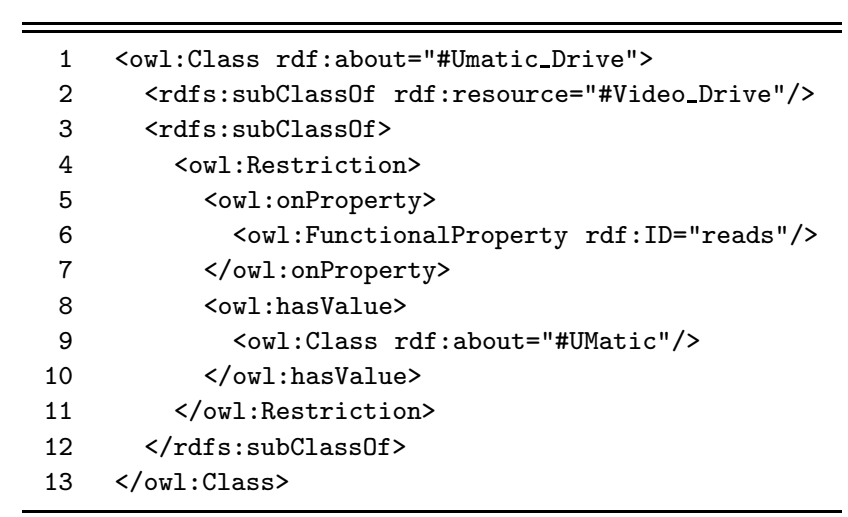

Table 6 Definition of the property is-Read-by as the inverse property of reads

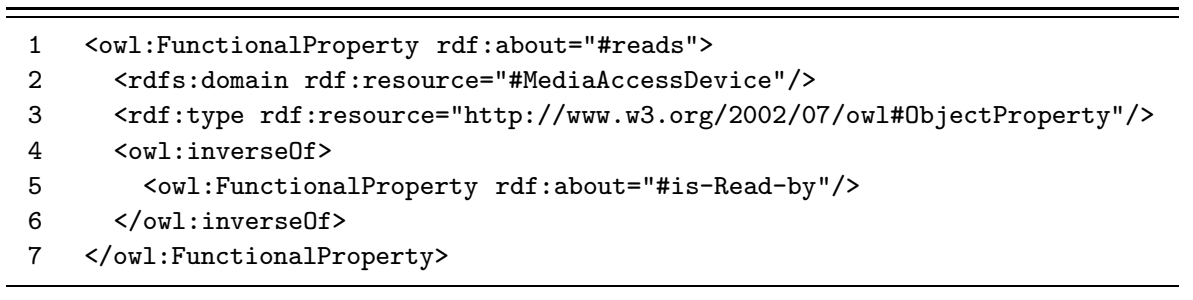

Table 7 Definition of cardinality constraints for the connects property

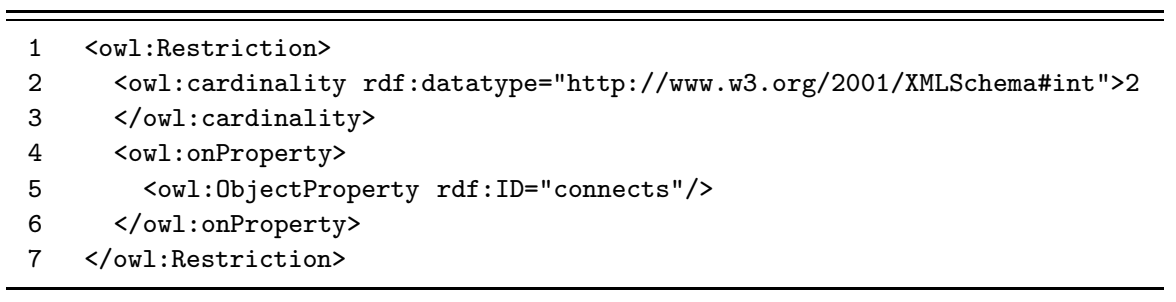

A similar restriction can be defined on the type of drive that is able to read a tape. Table 6 presents the use of the OWL construct owl: inverse0f to define such an inverse relation between properties. In this example, the property reads of the class MediaAcessDevice is defined as having an inverse property named is-Read-by.

Some properties of the defined ontology should not be allowed to have an arbitrary number of values. A NetworkLink is the concept used to characterise the connection between exactly two Network entities. Unless cardinality constrains are defined, OWL assumes that any instance of a class may have an arbitrary number of values for a given property. A restriction on the property connects was defined to assure that the concept is clearly defined (Table 7 ). 
3.3.1 Ontology Integration

The problem of data merging lies at the core of many metadata applications. The goal is to obtain a global view of a set of schemas developed independently. As there are many different type of models and applications, this problem has, many times, been tackled independently in specific domains. A model, being a formal description, can be a database schema, a UML model, a message format or an ontology.

Initial work in this area was carried out in the database scientific field $[36,37]$. However, the same aspects of data integration have been recently studied in the ontology domain. It is unrealistic to expect that all organizations and application systems will use a common, shared ontology because 1) even if a particular ontology becomes favoured, there will likely be many legacy solutions still in existence, which use an outmoded but still valid alternative, and 2) ontologies are usually aligned with a particular perspective. The need for ontology integration is then still a research area where some contributions have been made.

Although a lot of work has been going on in this area, the process of ontology matching requires, usually, human intervention. Most methodologies that have been proposed to implement this action are based on linguist approaches (like name or description similarities), inheritance hierarchy, cardinality matching or constraint-based approaches (e.g. on data types, value ranges, etc) [36,38-40]. Trying to improve this labor-intensive and error-prone process, some implementations, like GLUE [41], OntoBuilder [42] and oMAP [43], have been published with the purpose of providing semi-automatic matching tools. The accuracy of these solutions is however limited and its application is restricted to mapping ontologies within the same domain.

The ontology proposed in this paper provides an innovative approach by integrating concepts from multiple domains. This fact makes the process of using the semiautomated merging methodologies more difficult. Moreover, links between different domains have been defined during the conceptualization phase to enable an integrated view of managed resources and content. This cross-domain integration is not provided in any of the existing ontologies making the automated mapping approaches not helpful. Furthermore, as the main purpose of the proposed ontology is to hide much of the detail of the underlying metadata and to provide a business-driven management interface, only a small fraction of the metadata elements usually required to code each of the domains was considered.

The methodology used to define this ontology followed, nevertheless, some rules that are expected to simplify the integration mechanisms. As it can be seen from the examples presented on the conceptualization phase, the ontology tries to catch some concepts from existing schemas in both domains. This is clearly illustrated in the following tables that present the OWL code used to formalize some of the classes and properties of the proposed ontology.

Table 8 present an extract of the OWL code of the property RemainingManagedSpace of the class OnlineServer. As it can be seen, information introduced in the conceptualization phase is provided on the mapping proposal between these two concepts and the equivalent concepts in the CIM model. Formal semantics of this data integration is achieve by the definition of axioms that use the OWL construct equivalentClass or equivalentProperty.

An example of the mapping between multiple classes is illustrated in Table 9 where the classes Person and Rights are mapped into equivalent concepts in other description schemas. 
Table 8 Mapping between the property RemainingManagedSpace and the CIM equivalent concept

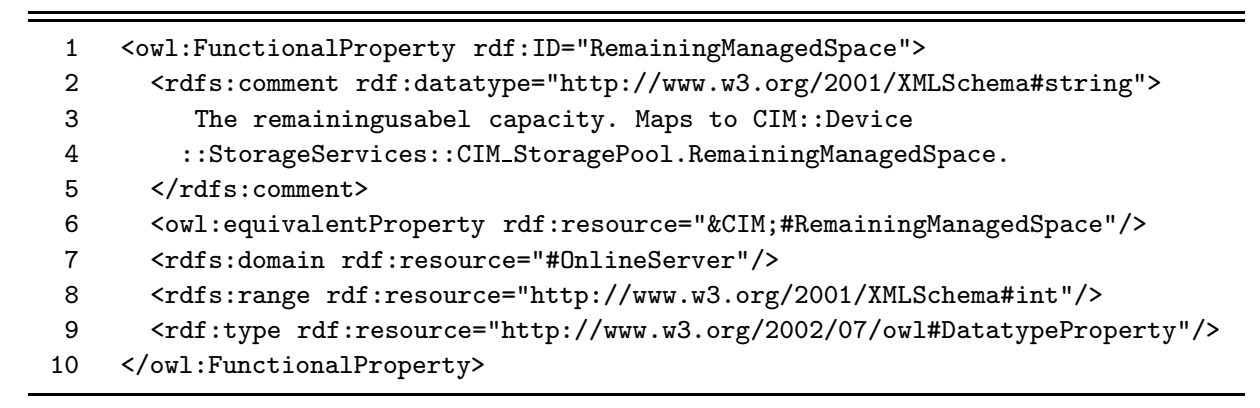

Table 9 Mapping between the classes Person and Rights with the equivalent concepts in TVA and MPEG7

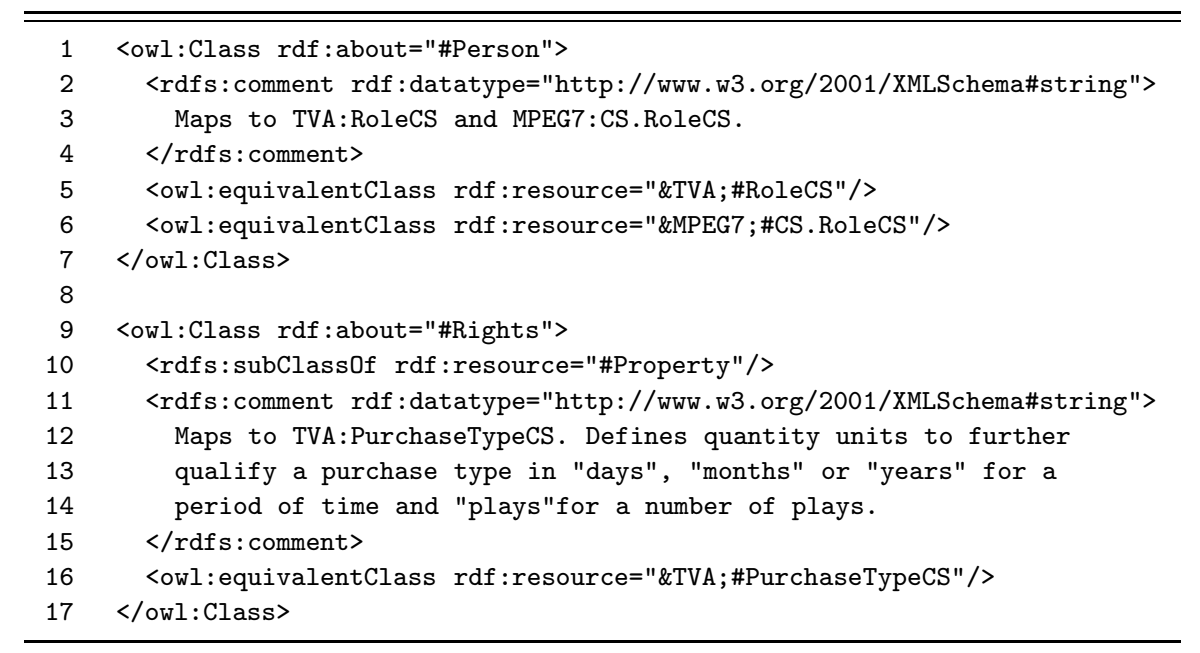

\section{Validation of the Semantic Management Model}

Validation of the ontology concepts was achieved in the broadcasting domain by populating the ontology with a set of instances for each of the defined classes. The architecture of the testbed includes a set of different archives that depend on the quality of the video:

- Full Quality Archive

- Browse Quality Archive

- Internet Archive

The Full Quality Archive keeps the original video copies in a format that can be used for a professional production of a new item. Due to processing, bandwidth and storage requirements, the regular use of this full quality material would imply the use of expensive equipment, making such a system unfeasible. To overcome this difficulty, 
a Browse Archive where lower quality copies of video are made available, through the network, to all the system workstations is available. Remote searching, previewing, selection and editing operations are then technically and economically feasible as no decoding hardware nor complex networks are required for working with a video quality still acceptable for these types of activities. Putting media assets in the Internet is a key factor for making information, which has an important cultural and economical value, known. The system enables the access to selected parts of the intranet archive in a very low quality copy. Search and manipulation facilities are reduced in this service.

Accordingly to its accessibility, the system includes three different archives:

- On-Line Archive

- Near-On-Line Archive

- Off-Line Archive

The facility of accessing video material decreases from the On-Line until the OffLine archive, making the slot of time needed since the user requests an item until he/she gets it, progressively larger. Remote operation of robotic datatapes enables the automatic transfer of material from the Near-On-Line to the On-Line Archive.

Figure 5 presents a partial view of the description of an instance of a programme ${ }^{1}$. Besides providing information concerning the characterization of the content, the different available versions are also identified together with the tapes where they are stored in: the Programme identified as Warriors of the Net is a Pre-Recorded stream whose target audience are Teenagers, has no rights associated (PlayForEver), is a ScienceFiction/Fantasy Programme and has a set of people participating having different roles (Narrator, InvitedGuest, Producer, Director); the content is available as full quality from Tape 1 which is a Umatic tape (Figure 6).

The availability of drivers to read this format is illustrated in Figure 7. A different view of the ontology (Figure 8) provides information about the availability of the broadcast quality version of this content (File2) from an Online Server (OnLineServer1) connected to Network1.

This example provides a clear view of the integration of content description and resource description metadata and of its applicability to solve e.g. 'Example 3'. From the illustrations of the ontology it is clear that the program Warriors of the Net can only be accessed through two available drivers able to read Umatic tapes.

This integrated view of the system components (both content and devices) enables the manager to have a unique view of all the information. From his point of view, information that globally describes content (like the genre of the film or the technical sheet) rather than parts of the content (splitting the program into scenes or having the indication of the exact timecode where a given situation occurs) is important. However, in order to enable him to automate some management procedures, this information has to be linked to description of the devices/components available in the system.

\section{Conclusions and Future Work}

The main objective of the work presented in this paper was to propose strategies that enable the effective deployment of complex networked audiovisual libraries. Given the

\footnotetext{
1 Instances of the ontology are presented in red while classes are pictured in brown.
} 


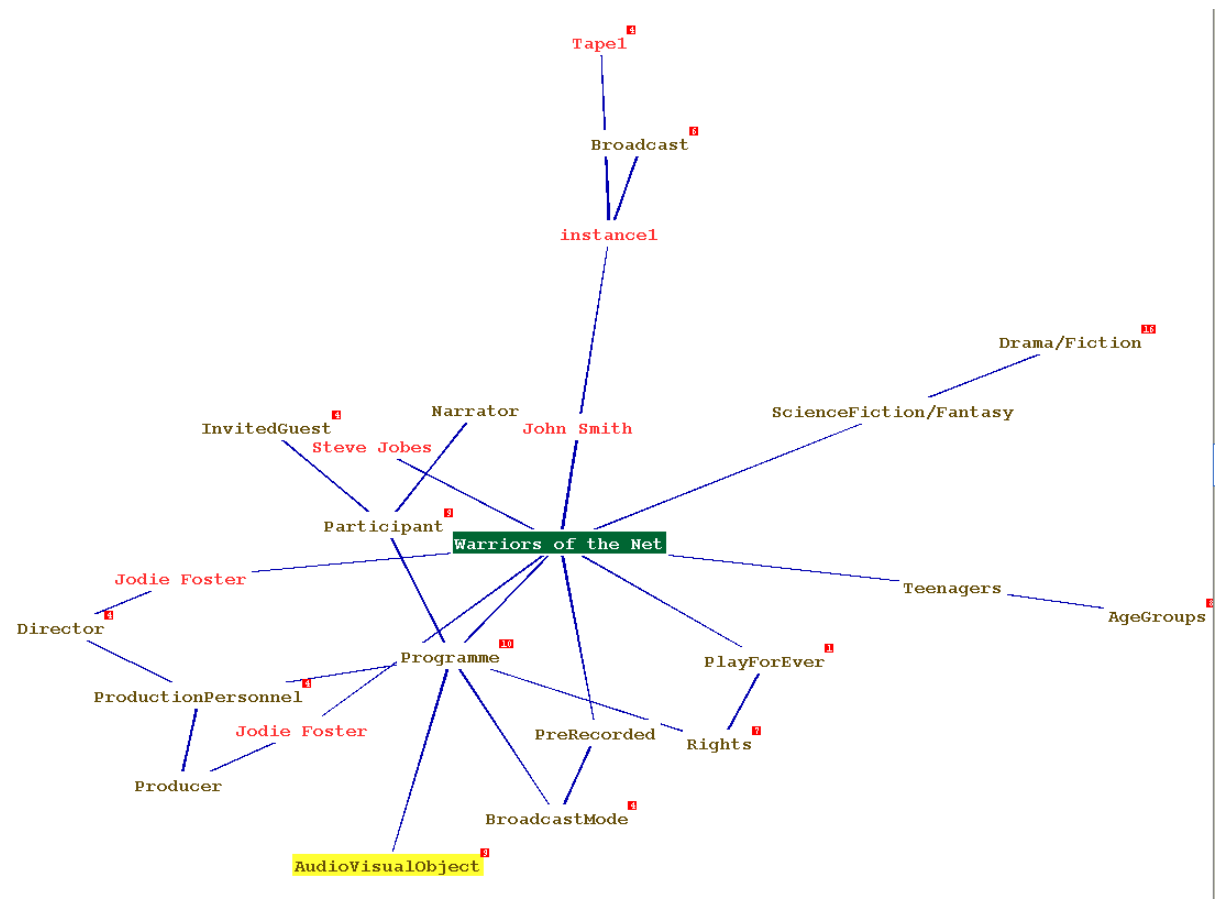

Fig. 5 Partial view of the description of a Programme instance (Warriors of the Net)

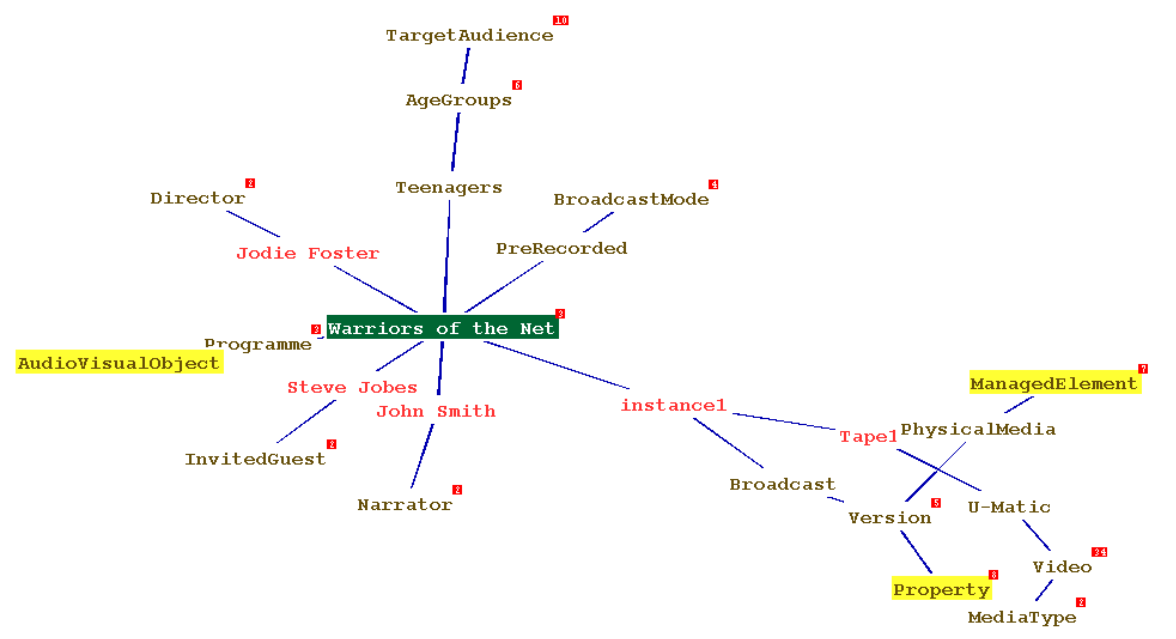

Fig. 6 Partial view of the description of a Programme instance (Warriors of the Net) - version of the material 


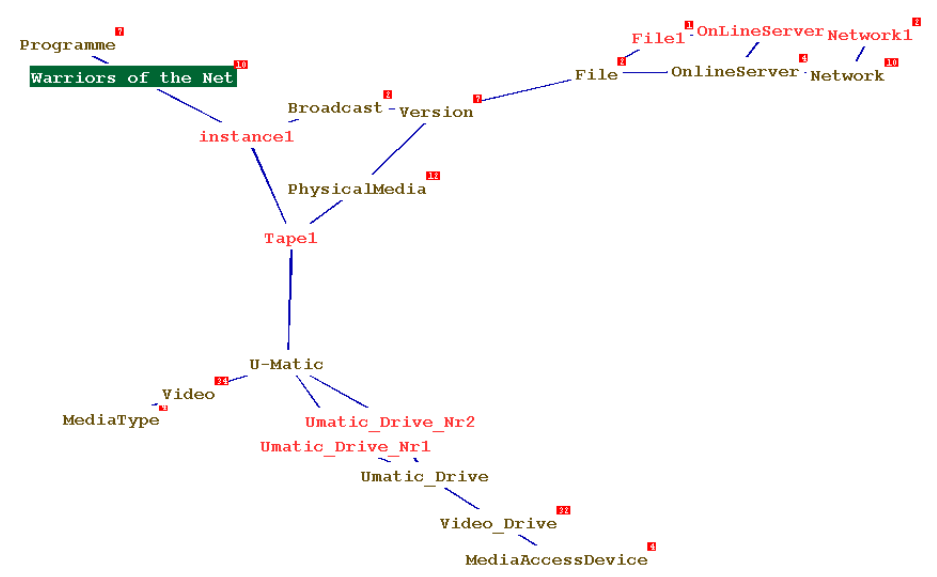

Fig. 7 Partial view of the description of a Programme instance (Warriors of the Net) - availability of video drivers able to read it

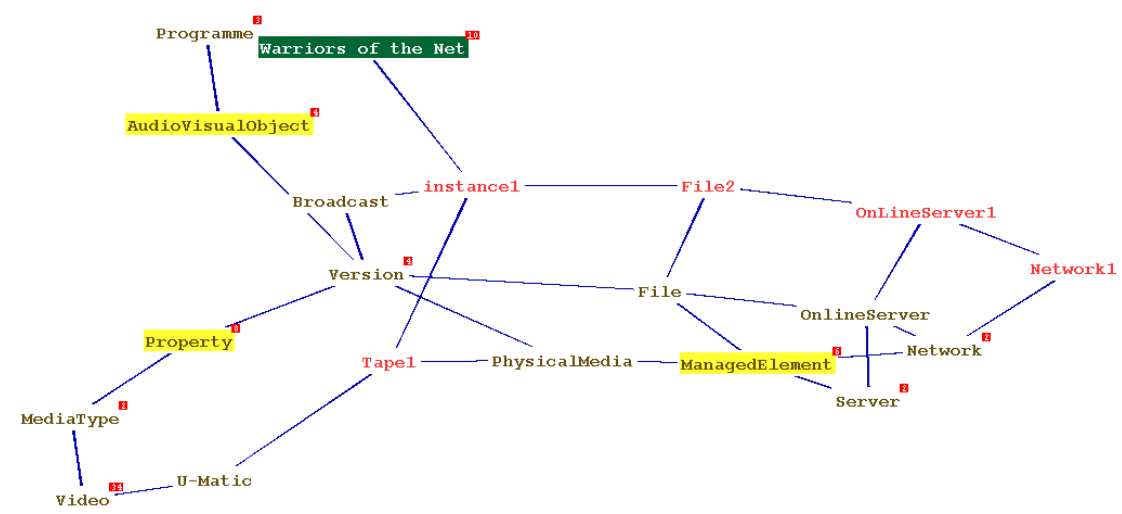

Fig. 8 Partial view of the description of a Programme instance (Warriors of the Net) - online availability

independent development of media description metadata schemas and device description metadata, the need for integrating these two domains was identified.

The definition and formalization of a domain ontology that inherits the main concepts from the existing description models but reduces the complexity and number of entities involved was presented. It combines concepts from different worlds allowing the integration of information but isolating management applications from the underlying details and then facilitating the definition of business or high-level decisions concerning the integrated management of complex audiovisual libraries.

The tests conducted using a prototype platform demonstrate that the manager can easily access important information that can help him/her on implementing crucial management tasks. This proves the important of this new approach.

Future topics for research include the use of other semantic web technologies to enable the description of services capable of implementing a set of management rules. 
For that we plan to use SWRL (Semantic Web Rule Language) that allows rules to be expressed in terms of OWL concepts and that can reason about OWL individual and OWL-S (Semantic Markup for Web Services) to describe services capable of implementing some of the rules formalized. As an example, the implementation of a service that automatically upgrades a list of tapes that should be considered for recycling can be based on knowledge inferred from the ontology through an SWRL rule: a new property "needsReclycling" can be defined based on information captured from the ontology concerning the number of devices available to read that format or on the number of times the tape was mounted, read or written. If material is in risk and contains crucial information (e.g. was awarded a prize - property has-award - or has an important participant - property hasParticipant), this rule can be refined to provide the property "needsUrgentReclycling" which uses extra information from the ontology. This new information inferred from the ontology using SWRL rules can be used to automate a recycling service. The coordination of OWL, SWRL and OWL-S could then contribute to the automation of some of the business driven media asset management processes.

\section{References}

1. FIAT/IFTA Documentation Commission, Minimum Data List (1992)

2. ISO/IEC, Multimedia Content Description Interface, ISO/IEC International Standard $15938(2002)$

3. José M. Martinez and Rob Koenen and Fernando Pereira,MPEG-7: The Generic Multimedia Content Description Standard, Part 1, IEEE Multimedia, 9, 2, 78-87 (2002)

4. José M. Martinez,MPEG-7: Overview of MPEG-7 Description Tools, Part 2, IEEE Multimedia, 9, 3, 83-93 (2002)

5. EBU, PMeta 2.0 Metadata Library, EBU Tech 3295-v2 (2007)

6. Richard Hopper, EBU Project Group P/Meta - Metadata Exchange Scheme, v1.0, EBU Technical Review, 290, April (2002)

7. SMPTE, Metadata Dictionary Structure, SMPTE Standard 335M-2001 (2001)

8. ETSI, Broadcast and On-line Services: Search, select, and rightful use of content on personal storage systems (TV-Anytime); Part 3: Metadata, TS 102 822-3-1:4 (2006)

9. DCMI, Dublin Core Metadata Initiative, http://dublincore.org

10. J.D. Case and M. Fedor and M.L. Schoffstall and J. Davin, Simple Network Management Protocol (SNMP), IETF, RFC 1157 (1990)

11. K. McCloghrie and M.T. Rose, Management Information Base for Network Management of TCP/IP-based internets: MIB-II, IETF, RFC 1213 (1991)

12. IEEE, Standard for Media Management Systems (MMS) Architecture, IEEE Standard No 1244.1 (2000)

13. DMTF, CIM Schema v2.17.1, January (2008)

14. W3C, XML Schema Part 0: Primer, Second Edition,http://www.w3.org/TR/xmlschema0/, October (2004)

15. W3C, Extensible Markup Language (XML) 1.0 (Fourth Edition), http://www.w3.org/TR/2006/REC-xml-20060816, August (2006)

16. W3C, RDF Vocabulary Description Language 1.0: RDF Schema, http://www.w3.org/TR/rdf-schema/,February (2004)

17. W3C, RDF Primer, http://www.w3.org/TR/rdf-primer/, February (2004)

18. W3C, OWL Web Ontology Language Overview, http://www.w3.org/TR/owl-features/, February (2004)

19. Grigoris Antoniou and Frank van Harmelen,Handbook on Ontologies in Information Systems, Web Ontology Language: OWL, 76-92, Springer-Verlag (2003)

20. Jane Hunter, Enhancing the Semantic Interoperability of Multimedia through a Core Ontology, IEEE Transactions on Circuits and Systems for Video Technology, 13, 1, 49-58 (2003)

21. Raphael Troncy, Integrating Structure and Semantics into Audio-Visual Documents,Proceedings of the 2th International Semantic Web Conference (ISWC2003), LNCS, 2870, 566-581, Springer Berlin (2003) 
22. Antoine Isaac and Raphael Troncy, Designing and Using an Audio-Visual Description Core Ontology. In Workshop on Core Ontologies in Ontology Engineering, CEUR Proceedings, $118(2004)$

23. Chrisa Tsinaraki and Panagiotis Polydoros and Stavros Christodoulakis, Interoperability Support for Ontology-Based Video Retrieval Applications, Third International Conference on Video and Image Retrieval, LNCS, 3115, 2050-2059, Springer Berlin (2004)

24. Chrisa Tsinaraki and Panagiotis Polydoros and Stavros Christodoulakis, Integration of OWL Ontologies in MPEG-7 and TV-Anytime Compliant Semantic Indexing, 16th International Conference (CAiSE 2004), LNCS, 3084, 398-413, Springer Berlin (2004)

25. Chrisa Tsinaraki and Panagiotis Polydoros and Fotis Kazasis and Stavros Christodoulakis, Ontology-Bases Semantics Indexing for MPEG-7 and TV-Anytime Audiovisual Content, Multimedia Tools and Applications, 26, 3, 299-325 (2005)

26. Robert Garcia and Òscar Celma, Semantic Integration and Retrieval of Multimedia Metadata, Proceedings of the 4th International Semantic Web Conference (ISWC2005), (2005)

27. Rosa Gil and Roberto Garcia and Jaime Delgado, An Interoperable Framework for IPR using Web Ontologies, Legal Ontologies and Artificial Intelligence Techniques (LOAIT 2005), IAAIL Workshop Series, Wolf Legal Publishers, 135-148 (2005)

28. Roberto Garcia and Rosa Gil and Jaime Delgado, A web ontologies framework for digital rights management, Artif. Intell. Law, 15, 2,137-154,Kluwer Academic Publishers (2007)

29. R. Garcia and F. Perdrix and R. Gil, Ontological infrastructure for a semantic newspaper, Proceedings of Semantic Web Annotations for Multimedia Workshop (SWAMM'06), World Wide Web Conference, (2006)

30. Richard Arndt, Raphal Troncy, Steffen Staab, Lynda Hardman and Miroslav Vacura, COMM: Designing a Well-Founded Multimedia Ontology for the Web, In 6th International Semantic Web Conference (ISWC'07), LNCS, 4825, 30-43 (2007)

31. Lawrence E. Menten, Experiences in the Application of XML for Device Management, IEEE Communications Magazine, 42, 7, 92-100 (2004)

32. Mi-Jung Choi and Hyoun-Mi Choi and James W. Hong amnd Hong-Taek Ju, IEEE Communications Magazine, 42, 7, 84-91 (2004)

33. Jorge E. López Vergara and Victor A. Villagró and Julio Berrocal, Applying the Web Ontology Language to Management Information Definitions, IEEE Communications Magazine, 42, 7, 68-74 (2004)

34. S. Quirolgico and P. Assis and A. Westerinen and M.Baskey and E. Stokes, Towards a Formal Common Information Model Ontology, Proceedings of The Fifth International Conference on Web Information Systems Engineering (WISE 2004), LNCS, 3307, 11-21, Springer Berlin (2004)

35. Jorge E. López Vergara and Victor A. Villagró and Julio Berrocal, On the Formalization of the Common Information Model Metaschema, 16th IFIP/IEEE International Workshop on Distributed Systems: Operations and Management (DSOM 2005), LNCS, 3775, 1-11, Springer Berlin (2005)

36. Erhard Rahm and Philip A. Bernstein, A Survey of approaches to automatic schema matching, The International Journal on Very Large Data Bases, 10, 4, 334-350, SpringerVerlag New York, Inc (2001)

37. Philip A. Bernstein, Applying model management to classical meta data problems, Proceedings of the 1st Biennial Conference on Innovative Database Research (CIDR), 209-220 (2003)

38. Jayant Madhavan and Philip Bernstein and Kuang Chen and Alon Halevy and Pradeep Shenoy, Corpus-based Schema Matching, Proceedings of the Workshop on Information Integration. 18th International Joint Conference on Artificial Intelligence, 59-66 (2003)

39. Jorge E. López Vergara and Victor A. Villagró and Julio Berrocal, An ontology-based method to merge and map management information models, Proceedings of the HP Openview University Association Tenth Plenary Workshop (HPOVUA03) (2003)

40. Jorge E. López Vergara and Victor A. Villagró and Julio Berrocal, Benefits of Using Ontologies in the Management of High Speed Networks, 7th IEEE International Conference on High Speed Networks and Multimedia Communications (HSNMC'04), LNCS, 3079,10071018,Springer Berlin (2004)

41. AnHai Doan and Jayant Madhavan and Pedro Domingos and Alon Halevy, Ontology Matching: A Machine Learning Approach, Handbook on Ontologies, International Handbooks on Information Systems, 385-403, Springer Verlag (2004)

42. Haggai Roitman and Avigdor Gal, OntoBuilder: Fully Automatic Extraction and Consolidation of Ontologies from Web Sources using Sequence Semantics, Proceedings of the 
International Conference on Semantics of a Networked World:Semantics of sequence and time dependent data (ICSNW 2006), LNCS, 4254, 573-576, Springer Berlin (2006)

43. Umberto Straccia and Raphael Troncy, oMAP: Combining Classifiers for Aligning Automatically OWL Ontologies, Proceedings of The 6th International Conference on Web Information Systems Engineering (WISE 2005), LNCS, 3806, 133-147, Springer Berlin (2005)

44. Paula Viana, Media asset management in broadcasting : new approaches to enable the effective management of physical resources and media objects, http://aleph.fe.up.pt/F/DSH4631BHVMUJMN7AVHF46Y6VUEADFLI5BPUTS8IR5YQKAD8GG23565 ? func $=$ full-set-set\&set_number $=006515 \&$ set_entry $=000001 \&$ format $=999, \mathrm{PhD}$ Thesis, Faculty of Engineering, University of Porto (2008)

45. ETSI, Broadcast and On-line Services: Search, select, and rightful use of content on personal storage systems (TV-Anytime);Part3:Metadata; Sub-part 1: Phase 1 - Metadata schemas, TS 102822-3-1 v1.3.1 (2006)

46. EBU, EBU System of Classification of RTV Programmes,EBU Tech ESCORT 2.4 (1995) 47. ETSI, Digital Video Broadcasting (DVB); Specification for Service Information (SI) in DVB systems, EN 300468 V1.3.1 (1998)

48. Marco Bertini and Alberto Del Bimbo and Carlo Torniai,Multimedia enriched ontologies for video digital libraries,International Journal of Parallel, Emergent and Distributed Systems, 22:6,407 416 (2007)

49. K. Petridis and S. Bloehdorn and C. Saathoff and N. Simou and S. Dasiopoulou and V. Tzouvaras and S. Handschuh and Y. Avrithis and Y. Kompatsiaris and S. Staab,Knowledge representation and semantic annotation of multimedia content, IEE Proceedings on Vision, Image and Signal Processing, 153, 3, 255-262, June (2006) 